\title{
Fonctions à croissance régulière et itération d'ordre fractionnaire
}

\author{
par Paul Lévy (à Paris)
}

CHAPITRE I.

\section{Notions générales sur les fonctions régulières.}

1. La notion de fonction à croissance régulière est une de ces notions intuitives qui semblent d'abord parfaitement claires, mais dont la difficulté apparaît à la réflexion; les remarques simples que l'on peut faire sur les raisons qui nous font considérer certaines fonctions comme régulières ne suggèrent en effet aucune définition précise de la régularité. Le présent travail contient l'exposé de recherches dont le but est d'obtenir une telle définition.

Je me suis laissé guider dans ces recherches par des considérations intuitives, et forcément subjectives, basées d'une part sur la notion esthétique de courbe régulière (c'est-à-dire sans sinuosités), d'autre part sur la notion d'induction, les remarques que l' on peut faire sur les exemples simples de courbes régulières semblant susceptibles d'être généralisées. Des considérations de cette nature ne peuvent être invoquées qu'au point de vue heuristique; il s'agit ensuite de démontrer les résultats que l'on a été eonduit à énoncer. Dans le cas particulier, j'ai ainsi été conduit à énoncer des rẻsultats que je n'ai pas réussi à démontrer; après quelques tentatives infructueuses, je crois utile, dans l'intérêt de la science, de faire comnaître tout de même l'ensemble de la théorie à laquelle j' ai été conduit. Le lecteur trouvera donc, à côté de certains résultats précis et démontrés, d'autres énoncés dont l'exactitude n'est que probable. Je serais heureux si cette lecture pouvait provoquer de nouvelles recherches, destinées à démontrer les résultats que je n'ai fait qu'énoncer: c'est un sujet de recherches difficile, mais même un résultat partiel ne serait pas sans importance.

Des résumés de mes recherches ont déjà été présentés à l'Académie des Sciences de l'Institut de France (1926 et 1927), au Congrès pour l'A vancement des Sciences de Poitiers (1926), et au Congrès Intermational des Mathématiciens de Bologne (i928). Enfin, sous le titre "Introduction à une théorie des fonctions 
à croissance réguliére $s, j$ 'ai publié dans le Jourmal de Mathématiques (1928) une étude sur les échelles complètes de croissance, étıde qui est liée aux recherches dont j'expose ici. le résultat; je rappellerai d'ailleurs ce qui est nécessaire pour éviter au lecteur d'avoir à se reporter au Mémoire en question.

2. Pour indiquer ce qui me paraît être un des caractères essentiels de la notion de régularité, je prendrai l' exemple d' une fonction telle que $e^{x}+\sin x$; nous la considérons comme irrégulière, parce qu'elle oscille indéfiument entre les fonctions plus simples $e^{x}+1$ et $e^{x}-1$; de même la fonction $e^{x}+e^{-x} \sin \log x$, malgré la lenteur et la petitesse de ses oscillations, est irrégulière, parce qu'elle oscille une infinité de fois entre les fonctions plus simples $2 \operatorname{ch} x$ et $2 \operatorname{sh} x$. Au contraire, l'une quelconque de ces fonctions plus simples, 2 ch $x$ par exemple, est régulière, parce qu'il n'existe aucune fonction plus simple qu' elle qui lui soit égale une infinité de fois, pour des valeurs de $x$ indéfiniment croissantes.

De ces premières remarques, nous ne pouvons pas déduire une définition; il semble même que nous ayons augmenté la difficulté du problème, car il doit être plus difficile de définir la plus ou moins grande simplicité (ou en d'autres termes la plus ou moins grande régularité) des fonctions que de définir la régularité parfaite qui caractérise les fonctions que nous appelons régulières (ou à croissance régulière). Il résulte pourtant de ces remarques une propriélé précise de l'ensenable des fonctions régulières, qui doit vérifier les deux conditions suivantes :

Condition a): si deux fonctions distinctes $f(x)$ et $g(x)$ appartiement à cet ensemble, leur différence est pour $x$. assez grand différente de zéro et d'un signe déterminé; l'une d'elles est alors supérieure à l'autre, pour $x$ assez grand; nous dirons qu' elle croit plus vite que l'autre.

Condition $b)$ : si une fonction $g(x)$ n'appartient pas a cet ensemble, on peut trouver au moins une fonction $f(x)$ de cet ensemble telle que la différence $f(x)-g(x)$ ne soit ni constamment positive, ni constamment négative, pour $x$ assez grand; cela revient à dire qu' on ne peut ajouter à l'ensemble aucune fonction nouvelle sans que la condition $a$ cesse d'être vérifiée.

Les ensembles vérifiant ces conditions constituent ce que $\mathfrak{j}$ 'appelle des échelles complètes de croissance; si la première condition est vérifiée, mais non la seconde, l'échelle est incomplète. Les échelles, complètes ou incomplètes, ont été étudiées d'une manière générale dans mon Mémoire cité plus haut.

On observe que s' $s^{\prime} l s^{\prime}$ agit de fonctions continues (et les fonctions régulières seront bien entendu des fonctions continues), l'énoncé de la condition $b$ peut 
se simplifier: il suffit d'écrire que les fonctions $f(x)$ et $g(x)$ sont une infinité de fois égales, pour des valeurs de $x$ indéfiniment croissantes.

L'existence d'échelles complètes est évidente, au point de vue idéaliste. L'ensemble des fonctions continues étant supposé bien ordonné, on n'a qu'à examiner toutes les fonctions l' une après l' autre et classer dans un ensemble $\mathcal{E}$ toutes celles qui peuvent l'être, c'est-à-dire qui croissent, soit plus vite, soit moins vite, que n'importe quelle fonction classée dans $\mathscr{E}$; l'ensemble $\mathscr{E}$ finalement obtenu sera une échelle complète.

On remarque que, chaque fois que dans l'application de ce procédé on obtient une fonction $f(x)$ acceptable, on peut soit la classer elle même dans l'ensemble $\mathcal{E}$, soit choisir, pour la classer dans $\mathcal{E}$, une autre fonction, égale une infinité de fois à $f(x)$, et acceptable, e' est-à-dire croissant, soit plus vite, soit moins vite, que n'importe laquelle des fonctions choisies initialement. De toute facon, l'ensemble ainsi formé sera une échelle complète.

3. Les fonctions régulières doivent donc constituer une échelle complète de croissance. Mais il s'agit de distinguer l'ensemble de ces fonctions des autres échelles complètes. Dans ce but, on peut d'abord se proposer de faire intervenir certains caractères simples des fonctions. On sait que des dérivations peuvent accentuer les irrégularités des fonctions; ainsi la fonction $\frac{1}{x}+\frac{\sin x}{x^{10}}$ est positive pour $x>0$ et chacune de ses 9 premières dérivées a un signe constant pour $x$ assez grand, mais la dixième a une infinité de changements de signes; c'est là une circonstance que nous considérons comme une preuve de l'irrégularité de cette fonction.

Une deuxième condition imposée à la définition de la régularité sera donc que les fonctions régulières soient continues, indéfiniment dérivables, chacune de leurs dérivées ayant un signe constant pour $x$ assez grand, c' est-à-dire à partir d'une certaine valeur de $x$ qui peut n'être pas la même pour toutes les dérivées. Ainsi la fouction $e^{-x^{2}}$ est régulière; sa dérivée de rang $n$ est, pour $x$ assez grand, du signe de $(-1)^{n}$; mais la valeur $x_{n}$ à partir de laquelle il en est ainsi augmente indéfiniment avẹc $n$; on ne peut pas trouver un intervalle déterminé daus lequel toutes les dérivées aient un signe constant, et soient par suite monotones $\left.{ }^{1}\right)$.

(1) On sait par un résultat de M. Serge Bernstein que, s'il en etait ainsi, la condition du texte entrainerait l'analyticité des fonctions considérées; mais le fait que les $x_{n}$ ne soient pas bornés empêche l'application de ce résultat. La question se pose de savoir si on peut le généraliser au cas qui nous occupe. Je considère d'ailleurs au'il n'est pas donteux que les 
Remarquons d'ailleurs que cette condition d'existence et de monotonie de toutes les dérivées, si elle est nécessaire, n' est sârement pas suffisante pour la régularité. Ainsi la fonction $e^{x}+\sin x$ est irrégulière, bien que toutes ses dérivées soient positives pour $x$ positif; de même la fonction

$$
\frac{1}{x \log x}\left(1+\frac{1}{2} \sin \log \log x\right)
$$

a chacune de ses dérivées monotones pour $x$ assez grand et est irrégulière.

Observons que cette condition de monotonie, jointe à la précédelite, domne un résultat positif: tous les polynomes sont des fonctions régulières. Si en effet un polynome $P(x)$ de degré $n$ n'était pas régulier, on devrait trouver une fonction régulière $f(x)$ égale une infinité de fois à ce polynome; cela n'est pas possible, le théorème de RoLle nous montrant que la dérivée d'ordre $n+1$ de $f(x)$ devrait changer de signe une infinité de fois.

Nous avons dit tout à l'heure qu' une fonction nous apparait comme parfailement régulière si on ne peut pas en trouver une plus régulière qui lui soit égale une infinité de fois; dans le cas des polynomes, cet énoncé prend un sens très précis.

4. L'insuffisance de la propriété précédente, et l'absence de toute autre opération de l'analyse élémentaire qui soit plus efficace que la dérivation pour déceler les irrégularités des fonctions, conduit à introduire des considérations d'un tout autre ordre, basées sur la nature des opérations analytiques qui conduisent à définir les fonctions considérées. All lieu de chercher à mettre en évidence une propriété intrinsèque des fonctions qui pourrait caractériser la régularité, nous sommes conduits à former de proche ell proche de nouvelles fonctions régulières par des opérations analytiques qui ne soient par ellesmêmes susceptibles d'introduire aucune irrégularité; de telles opérations sont ce que nous appellerons des opérations régulières. En partant des polynomes, on pourra par ces opérations former des eusembles étendus de fonctions régulières.

Ces opérations régulières doivent comprendre all moins la dérivation, l'intégration, les quatre opérations élémentaires, et, dans le cas d' une fonction $y=f(x)$ indéfiniment croissante, la formation de la fonction inverse $x=f_{-1}(y)$

fonctions régulières soient analytiques; mais cela ne résulte peut-être pas nécessairement des conditions déjà indiquées.

Il est probable qu' inversement, toute fonction analytique, réelle pour $x$ très grand, et méromorphe à l'infini, est régulière. Mais les fonctions ayant à l'infini un point singrulier essentiel peuvent être régulières on irrégulières. 
et des fonctions régulières de $f(x)$. Ces opérations et leurs combinaisons sont ce que nous appellerons les opérations régulières élẻmentaires.

Par ces opérations répétées un nombre fini $n$ de fois, en partant d'un polynome de degré $p$, on ne peut obtenir que des fonctions dépendant d'une manière continue d' un nombre fini de paramètres; même en augmentant indéfiniment $n$ et $p$, même en définissant de nouvelles opérationș régulières, on ne peut pas arriver a former ainsi toutes les fonctions régulières. On n' obtiendra sûrement pas un ensemble contenant des fonctions plus rapidement croissantes que n'importe quelle fonction donnée. Pourtant déjá une question se pose: la troisième condition ainsi imposée à la définition de la régularité est-elle compatible avec les précédentes; est-on notamment assuré que deux fonctions distinctes formées par des opérations régulières ne peuvent pas être une infinité de fois égales?

La réponse ne semble pas douteuse; il semble même que l'on puisse arriver à une démonstration par une classification méthodique des divers infiniment petits ou infiniment grands introduits par les opérations indiquées, et par des développements en séries de forme suffisamment générale. Mais la grande variété des combinaisons à prévoir rend la démonstration rigoureuse difficile. Disons donc seulement, pour résumer les résultats obtenus jusqu'ici, que des considérations intuitives nous ont conduit à penser qu' il était possible de définir un ensemble de fonctions régulières vérifiant les conditions suivantes:

1) Cet ensemble constitue une échelle complète de croissante.

2) Toutes les fonctions de cet ensemble sont continues, indéfiniment dérivables, et sont monotones pour $x$ assez grand; (la monotonie des dérivées résulte alors de la condition suivante).

3) Cet ensemble est fermé par rapport aux opérations régulières élémentaires définies ci-dessus.

5. Il est essentiel de définir d'autres opérations régulières; les résultats principaux de ce travail reposeront sur l'étude de l'itération régulière. Mais quelques remarques préalables seront utiles.

Considérons une fonction de deux variables $f(x, y)$, et supposons que, pour $y$ constant, ce soit une fonction régulière de $x$, et que d'autre part elle dépende d'une manière continue de $y$; il $\mathrm{y}$ a lieu de penser qu' on peut la considérer comme régulière en $x$ et $y$, et que par suite une fonction de la forme $f[\varphi(t), \psi(l)]$ est régulière en $t$ si $\varphi(l)$ et $\psi(t)$ sont des fonctions régulières indéfiniment croissantes de $t$; si cette propriété est vérifiée, nous dirons que $f(x, y)$ est une fonclion régulière des deux variables $x$ et $y$. Pour montrer 
que cette régularité par rapport aux deux variables résulte bien de la régularité par rapport à chacune des variables, il suffit d'établir que la régularité par rapport à chacune des variables entraine celle de $f(x, x)$; en appliquant ce résultat à la fonction $F(t, u)=f[\varphi(t), \psi(u)]$, on obtient le résultat plus général concernant la régularité de $f[\varphi(l), \psi(t)]$,

La dérivation et l'intégration d'une fonction de deux variables doivent alors être ajoutées a la liste des opérations régulières. Observons notamment qu' une intégrale du type

$$
f(x)=\int_{0}^{\infty} F(x, y) \varphi(y) d y
$$

doit être une fonction régulière de $x$ si $F(x, y)$ et $\varphi(y)$ sont des fonctions régulières.

Il faut aussi admettre la régularité de chaque branche de fonction définie par l'équation implicite $f(x, y)=0$, si la fonction $f(x, y)$ est régulière. Il en résulte que toute détermination, réelle pour $x$ assez grand, d'une fonction algébrique, est régulière; il en est donc de même pour toute fonction algébrique de $x, e^{x}, \log x$.

Il faut d'autre part éviter d'étendre aux séries la remarque faite sur l'intégrale (1). Ainsi dans la série

$$
e^{x}=1+\frac{x}{1}+\ldots+\frac{x^{n}}{n !}+\ldots
$$

ainsi que dans les séries que l'on en déduit en ne conservant que les termes de rangss multiples d'un entier $p$, le terme de rang $n$, soit $\frac{x^{n p}}{(n p) !}$, dépend d'une manière régulière de $x$ et de $n$; pourtant la somme, régulière pour $p=1$ ou 2 , est irrégulière pour $p>2$; pour $p=4$, par exemple, elle a la valeur $\frac{\operatorname{ch} x+\cos x}{2}$.

La raison en est facile à comprendre; dans la série (2), $x$ croissant, le rang du plus grand terme croît; chaque terme joue à son tour un rôle prépondérant; les différents termes déterminent successi vement l'ordre de grandeur de la fonction comme les poteaux successifs d' une ligne télégraphique soutiennent cette ligne, qui s' abaisse chaque fois entre deux poteaux. On a done des irrégularités d'autant plus marquées que les terines conservés sont plus espacés, même si leurs rangs forment une suite régulière. 
Du moins, l'image qui précède serait exacte si l'on prenait les termes de raugs $n=1,2^{2}, \ldots, p^{2}, \ldots$, ou d' une suite de nombres croissant plus rapidement eucore. En prenant des termes dont les rangs constituent une progression arithmétique, on a un nombre croissant (comme $\sqrt{x}$ ) de termes qui sont sensiblement du même ordre de grandeur, et le cas simple ou il n'y a qu'un terme prépondérant (ou un petit nombre de tels termes) n'est pas réalisé. L'irrégularité de la série n' est pas certaine dans ces conditions; mais la remarque que dans la série $\iota^{x}$, en prenant la somme des termes de $p$ en $p$, on obtient une fonction régulière si $p=1$ oll 2 et irrégulière si $p=3$ ou 4 montre bien la difficulté d'une théorie générale. Retenons seulement qu'on ne sera pas certain a priori, quelle que soit la régularité de la formation des termes d'une série, que sa somme soit régulière, et souvent on établira au contraire aisément qu' elle est irrégulière; au contraire, dans le cas d'une intégrale, qui n'a pas la même cause de discontinuité, on est sûr de la régularité.

6. Il existe un lien évident entre la notion de fonction régulière et celle d'une suite régulière de nombres $y_{1}, y_{2}, \ldots, y_{n}, \ldots ;$ si une fonction $f(x)$ est régulière, la suite des nombres $y_{n}=f(n)$ est régulière, et sa domnée détermine parfaitement la fonction $f(x)$, puisqu'il est impossible que deux fonctions régulières distinctes soient égales pour toutes les valeurs entières de $x$; la détermination de $f(x)$ connaissant les nombres $y_{n}=f(u)$ est ce que nous appellerons l'interpolation régulière de cette suite de nombres.

Il n'est guère douteux que toute suite qui n'est pas régulière oscille indéfiniment entre deux suites régulières bien déterminées, ou, ce qui revient au même, entre les fonctions correspondantes.

Par une extension naturelle de la notion d'opération régulière, on peut admettre que tonte formule permettant de définir une suite, si elle n'introduit aucune cause d'irrégularité, ne peut définir qu'une suite régulière. Il doit en être ainsi notamment dans le cas d' une relation de récurrence de la forme

$$
y_{n}=\varphi\left(n, y_{n-1}\right)
$$

si la function $\varphi(x, y)$ est régulière. On obtient alors une suite régulière dont l'interpolation régulière définit ane fonction bien déterminée $f(x)$. Il est important d'observer que, bien que cette interpolation puisse être défnie indépendamment de la relation de récurrence (3), la fonction obtenue vérifie sârement cette relation étendue an cas des valeurs non entières de $x$, c'est-à-dire que l'on a

$$
f(x)=\varphi[x, f(x-1)]
$$


Les deux membres sont en effet des fonctions régulières, égales pour toutes les valeurs entières de $x$; ils sont donc égaux.

Ce principe a de nombreuses applications. Il suffit de mentionner le problème de l'interpolation régulière des sommes successives d'une série, et l'étude de la fonction eulérienne qu'on peut déduire d'une telle interpolation. Nous l'appliquerons au cas où l'on donne une fonction régulière $f(x)$, monotone et supérieure à $x$; la suite des nombre itérés

$$
x_{0}, \quad x_{1}=f\left(x_{0}\right), \ldots, \quad x_{n}=f\left(x_{n-1}\right)=f_{n}\left(x_{0}\right), \ldots,
$$

est une suite régulière de nombres indéfiniment croissants; l'interpolation régulière de cette suite permet de définir une fonction régulière $f_{x}\left(x_{0}\right)$ d'une variable $\alpha$, et, bien que cette fonction puisse être obtenue sans tenir compte des relations de récurrence (4), élle vérifie sûrement la relation

$$
f_{\alpha+1}\left(x_{0}\right)=f\left[f_{\alpha}\left(x_{0}\right)\right]
$$

qui généralise les relations (4).

Il faut d'ailleurs observer que le raisonnement qui précède n' est utile que pour établir, au point de vue idéaliste, l'existence de la fonction itérée régulière $f_{x}\left(x_{0}\right)$. Les déterminations effectives de cette fonction reposeront au contraire sur la relation de récurrence (5) et sur une étude asymptotique qui permettra de distinguer des autres la solution régulière de celte équation.

CHAPITRE II.

\section{L'itération régulière.}

\%. Pour l'étude de l'itération, nous supposerons essentiellement la fonction $f(x)$ étudiée continue, monotone, et croissant indéfiniment avec $x$, et de plus soit constamment supérieure, soit constamment inférieure à $x$; l'un de ces cas se ramenant à l'autre par les relations entre les fonctions inverses, nous supposerons pour fixer les idées $f(x)>x$. Donc, en définitive, pour $x$ supérieur à une certaine valeur $a$, la fonction $f(x)$ est continue, croissante, et supérieure à $x$.

Le problème de l'itération consiste dans la recherche d'une fonction $f_{x}(x)$ vérifiant l'équation fonctionnelle

$$
f_{\alpha+\beta}(x)=f_{\beta}\left[f_{\alpha}(x)\right],
$$

et se réduisant à $f(x)$ pour $\alpha=1$; il en résulte évidemment que pour $\alpha$ 
entier, $f_{x}(x)$ se réduit aux itérées d'ordres entiers bien définies par la formule de récurrence

$$
f_{n+1}(x)=r\left[f_{n}(x)\right]
$$

Nous imposerons de plus à la fonction $f_{x}(x)$ d'être continue et croissante aussi bien par rapport $\dot{a} x$ que par rapport à $\alpha$. Enfin nous supposerons toujours $x>a$ et $\alpha>0$ (le cas où $\alpha$ est négatif se traitant ensuite sans difficulté, puisque $f_{\alpha}$ et $f_{-\alpha}$ sont des fonctions inverses l'une de l'autre).

On peut se placer à deux points de vue différents selon qu' on considère ou non a comme une variable. Malgré la plus grande portée de la première méthode, dont le principe a déjá été indiqué à la fin du Chapitre I, nous présenterons d'abord quelques remarques relatives au cas où l'on ne considère pas $\alpha$ comme une variable, et où l'on déduit $f_{\alpha}(x)$ des déterminations successives des itérées d'ordres $\frac{1}{2}, \frac{1}{4}, \frac{1}{8}, \ldots$, etc.

8. L'itérée d'ordre $\frac{1}{2}, f_{\frac{1}{2}}(x)=g(x)$, doit être solution dẹ l' équation fonctionnelle

$$
g[g(x)]=f(x)
$$

qui est un cas particulier de l'équation (6). Montrons d'abord qu'il existe une infinité de solutions de cette équation.

Choisissons une valeur $x_{0}$ de $x$, et pour $y_{0}=g\left(x_{0}\right)$ une valeur quelconque entre $x_{0}$ et $x_{1}=f\left(x_{0}\right)$. Nous pouvons prendre pour $g(x)$, dans l'intervalle $\left(x_{0}, y_{0}\right)$ une fonction continue, croissante, prenant aux extrémités de cet intervalle les valeurs $y_{0}=g\left(x_{0}\right)$ et $x_{1}=g\left(y_{0}\right)$, et à cela près quelconque. Si $y=g(x)$, la relation (7) donne $g(y)=f(x)$, et cette relation détermine successivement $g(x)$ dans les intervalles $\left(y_{0}, x_{1}\right),\left(x_{1}, y_{1}\right),\left(y_{1}, x_{2}\right), \ldots$; on a ainsi une fonction toujours continue et croissante, solution de l'équation (7). On voit qu' on peut la choisir arbitrairement dans une demi-période, en appelant période l'intervalle qui sépare $x$ et $f(x)$, ou encore $f_{\alpha}(x)$ et $f_{x+1}(x)$, c'est-à-dire l'intervalle pendant lequel l'indice d'itération augmente d'une unité.

Considérons deux solutions distinctes $g_{1}(x)$ et $g_{2}(x)$ de l'équation (7): il est impossible que $l^{\prime}$ une d' elles soit constamment supérieure à l'autre; si en effet on avait $g_{1}(x)>g_{2}(x)$, on aurait.

$$
g_{1}\left[g_{1}(x)\right]>g_{2}\left[g_{2}(x)\right]
$$

ce qui est impossible, les deux membres devant être égaux a $f(x)$; d'une 
manière plus précise, si pour $x=x_{0}$

$$
y_{0}=g_{1}\left(x_{0}\right)>z_{0}=g_{2}\left(x_{0}\right)
$$

dans l' intervalle $\left(z_{0}, y_{0}\right)$ on a

$$
g_{1}(x)<g_{1}\left(y_{0}\right)=f\left(x_{0}\right)=g_{2}\left(z_{0}\right)<g_{2}(x),
$$

et pour $x_{1}=f\left(x_{0}\right), g_{1}(x)$ est de nouveau supérieur à $f(x)$. Le signe de la différence $g_{1}(x)-g_{2}(x)$ change donc au moins deux fois par période et se reproduit périodiquement. Il résulte de ces remarques qu' il y a au plus une détermination régulière de $g(x)$.

La condition nécessaire et suffisante pour l'existence de cette itérée régulière d'ordre $\frac{1}{2}$ est d'ailleurs que la fonction dommée $f(x)$ soit elle-même régulière. La nécessité de cette condition est évidente, d'après la formule (7), une fonction régulière de fonction règulière étant elle-même régulière. La réciproque est plus délicate, et je n'ai pas pu en obtenir de démonstration rigoureuse. Observons seulement que la formule (7) définit une conpure dans l'ensemble des fonctions régulières, les fonctions $\varphi(x)$ telles que $\varphi[\varphi(x)]$ croît plus vite que $f(x)$ étant nu-dessus de la conpure et celles dont l'itérée croit moins vite étant au-dessous; c'est de l'étude des coupures dans les échelles de croissance, dont on trouvera les éléments dans mon mémoire déjà cité, que l'on peut espérer déduire une démonstration de l'existence de la fonction $g(x)$, indépendante de l'étude de $f_{\alpha}(x)$ considérée come fonction de $\alpha$.

Après avoir défini l'itérée d'ordre $\frac{1}{2}$, on définit successivement de la même manière les itérées d'ordres $\frac{1}{4}, \frac{1}{8}$, et ainsi de suite; on introduit ainsi de nouvelles fonctions choisies arbitrairement dans un quart, puis un huitième de période, et ainsi de suite. Tout compte fait, la détermination compléte de $f_{a}(x)$ dépend d'une fonction choisie arbitrariement dans une période; mais il n'y a qu' une fonction $f_{x}(x)$ qui soit régulière par rapport à $x$.

9. Plaçons-nous maintenant au second point de vue, qui consiste à considérer $\alpha$ comme variable. Désignant par $x_{0}$ une valeur particulière de $x$, nous poserons $x=f_{\alpha}\left(x_{0}\right)=\varphi(\alpha)$; la formule (6) prend alors la forme

$$
\varphi(\alpha+\beta)=f_{\beta}(x)
$$

et nous montre que la connaissance de $\varphi(\alpha)$ détermine complètement $f_{\alpha}(x)$. 
Si d'ailleurs on ne connait pas $f(x)$, on peut prendre pour $\varphi(\alpha)$, de zéro â l'infini, n' importe quelle fonction continue, monotone, et indéfiniment croissante; la formule (8), où $x=\varphi(\alpha)$, définit alors une fonction $f_{\beta}(x)$ solution de l'équation fonctionnelle (6), et qui est l'itérée de la fonction $f(x)$ obtenue en faisant $\beta=1$. Si au contraire cette fonction $f(x)$ est donnée, la fonction $\varphi(\alpha)$ ne pent être choisie arbitrairement que de $0 \dot{a} 1$, les valeurs extrêmes $x_{0}=\varphi(0)$ et $x_{1}=\varphi(1)$ devant être liées par la relation $x_{1}=f\left(x_{0}\right)$. Les valeurs de $\varphi(\alpha)$ pour $x>1$ résultent alor's de la formule

$$
\varphi(\alpha+n)=f_{n}[\varphi(\alpha) \mid
$$

oǹ $n$ est $u$ entier positif quelconque. L'itération d' une fonction $f(x)$ donnée ne dépend donc bien que d'une fonction choisie arbitrairement dans une période.

Nous désignerons par $\alpha=\lambda_{x}(y)$, et appellerons indice d'ilération ou logarithme d'ilèration de $y$ par rapport à $x$, le nombre $\alpha$ tel que $y=f_{\alpha}(x)$; si $y>x>\alpha$, e' est un nombre positif bien déterminé. Les logarithmes d'itération de $x$ et $y$ par rapport à $x_{0}$ seront désignés par $\lambda(x)$ et $\lambda(y)$, sans indice inférieur; $\lambda(x)$ est la fonction inverse de $\varphi(\alpha)$, et de même $\alpha=\lambda_{\infty}(y)$ et $y=f_{\alpha}(x)$ sont des fonctions inverses l'une de l'autre, $x$ étant constant et l'inversion portant sur $\alpha$ et $y$. Avec ces notations, l' équation fonctionnelle (6) prend la forme.

$$
\lambda_{x}(y)=\lambda(y)-\lambda(x),
$$

et nous voyons d'une manière plus claire encore que le problème de l'itération se ramène à la détermination de la seule fonction $\lambda(x)$, inverse de la fonction $\varphi(\alpha) ; f(x)$ étant donné, $\lambda(x)$ doit croître d'une manière continue de $\lambda\left(x_{0}\right)=0$, à $\lambda\left(x_{1}\right)=1$, et est à cela près quelconque de $x_{0}$ à $x_{1}$; pour $x>x_{1}$, cette fonction est bien déterminée par la formule

$$
\lambda\left[f_{n}(x)\right]=n+\lambda(x),
$$

qui équivaut à la formule (9).

Comparons deux déterminations différentes de la fonction itérée, déduites de deux déterminations $\varphi(\alpha)$ et $\psi(\alpha)$ de $f_{\alpha}\left(x_{0}\right)$; pour les valeurs entières $n$ de $\alpha$, ces deux fonctions sont égales à $x_{n}=f_{n}\left(x_{0}\right)$, et par suite égales entre elles; l'une de ces fonctions au plus peut donc être régulière. Leur irrégularité relative est d'ailleurs mieux mise en évidence quand on compare les fonctions inverses $\lambda(x)$ et $\mu(x)$; elles augmentent simultanément d'une unité quand on passe de $x$ á $f(x)$; il en résulte que la différence $\lambda-\mu$ est une fonction périodique aussi bien de $\lambda$ que de $\mu$; le rapport $\frac{d \lambda}{d \mu}$ varie aussi périodiquement: 
si de $x_{0}$ à $x_{1}$ on a pris pour $\lambda(x)$ et $\mu(x)$ des fonctions dont les dérivées ont une limite inférieure positive et une limite supérieure finie, on est donc assuré que $\frac{d \lambda}{d \mu}$ est, de $x_{0}$ à l'infini, borné inférieurement et supérieurement. Si $x$ et $y$ croissent indéfiniment, la partie entière de $\lambda(y)-\lambda(x)$ étant bien entendu indépendante du choix de la fonction itérée, la partie fractionnaire de cette différence a aussí son ordre de grandeur indépendant de ce choix.

Il existe donc au plus une itérée régulière, définie par une fonction régulière $\varphi(\alpha)$. Observons d'ailleurs que si la fonction $\varphi(\alpha)$ est régulière, la fonction $f_{\beta}(x)$ est régulière aussi bien par rapport à $x$ que par rapport $\dot{a} \beta$ ou par rapport à l'ensemble des deux variables; cela résulte immédiatement de la formule (8), qui peut s'écrire

$$
f_{\beta}(x)=\varphi\left[\beta+\lambda\left(x_{i}\right]\right.
$$

la fonction $\alpha=\lambda(x)$ étant régulière en même temps que la fonction inverse $\varphi(\alpha)$. Donc, sans admettre rien d'autre que la conservation de la régularité par une partie des opérations régulières élémentaires indiquées au $10^{\circ} 4$, nous sommes assurés que la régularité de $\varphi(\alpha)$ entraîne celle de $f_{\alpha}(x)$ aussi bien par rapport à $\alpha$ que par rapport à $x$, et celle de fonctions telles que $f_{x}(x)\left({ }^{1}\right)$. Nous sommes arrivés ainsi à un résultat plus complet qu'au n. ${ }^{\circ}$ précédent, où nous ne savions pas que l'itérée régulière en $x$ était anssi régulière en $\alpha$

Il est d'ailleurs toujours bien entendu que la condition nécessaire et suffisante pour l'existence de cette itérée régulière est la régularité de la fonction $f(x)$. Elle est nécessaire, la régularité de $f_{x}(x)$ impliquant celle de $f_{1}(x)=f(x)$; elle est suffisante, la fonction $\phi(\alpha)$ résultant comme nous l'avons dit au $10^{\circ} \dot{6}$ de l'interpolation régulière de la suite des $x_{n}=f_{n}\left(x_{0}\right)$, et la fonction $f_{\beta}(x)$, que l'on en déduit par la formule (8) ou la formule équivalente (12), étant régulière.

Il faut toutefois observer que cette réciproque, comme par la méthode du $n .^{\circ} 8$, repose sur des considérations non rigoureuses; même si l'on admet la compatibilité des conditions du n. 4 imposée à la définition de la régularité,

(1) Si $f(x)$ est une fonction rapidement croissante, cette fonction $f_{x}(x)$ est une fonction régulière croissant plus rapidement que tous les $f_{n z}(x)$. Ce point est important, la fonction

$$
\frac{f_{1}(x)}{f_{1}(1)}+\frac{f_{2}(x)}{f_{2}(2)}+\ldots+\frac{f_{n}(x)}{f_{n}(n)}+\ldots
$$

donné par P. ou Bors Reymond et M. E. Borex comme exemple de fonction eroissant plus rapidement que tous les $f_{n}(x)$ n'étant évidemment pas régulière. 
le résultat énoncé an $n .^{\circ} \mathrm{C}$ concernant la possibilité de réaliser l'interpolation régulière des suites régulières, en particulier de la suite des nombres $x_{n}=f_{n}\left(x_{0}\right)$, n'est qu'un résultat probable pour des raisons intuitives; on peut le considérer comme une quatrième condition imposée à la définition de la régularité, et dont l'exactitude sera à vérifier lorsque l'on proposera une définition précise.

10. Des considérations idéalistes établissant l'existence d'une itérée régulière, même si elles étaient rigoureuses, ne peuvent dispenser d'indiquer des formules qui permettent de définir effectivement cette fonction. Nous allons indiquer ces formules, obtenues en utilisant d'une part la relation fonctionnelle fondamentale, d'autre part une expression asymptotique des fonctions considérées, permettant de distinguer des autres fonctions considérées celle qui est régulière à l'infini; ces formules s'appliqueront d'ailleurs non seulement aux fonctions parfaitement régulières, mais à celles qui ne seront pas trop irrégulières. Il y aura plusieurs cas à distinguer, suivant la rapidité de la croissance de $f(x)$. Dans tous les cas, nous prendrons la relation fonctionnelle de l'itération sous la forme

$$
\alpha=\lambda(y)-\lambda(x)=\lambda\left(y_{n}\right)-\lambda\left(x_{n}\right),
$$

où $x_{n}=f_{n}(x), y_{n}=f_{n}(y)$. Il s'agit de définir $\alpha$, et comme $x_{n}$ et $y_{n}$ augmentent indéfiniment avec $n$, on peut $y$ arriver par des formules asymptotiques $\left({ }^{4}\right)$.

Supposons d'abord $f(x)$ de la forme

$$
f(x)=x+\omega(x)
$$

$\omega(x)$ étant une fonction positive, dont la dérivée $\omega^{\prime}(x)$ tende vers zéro. On remarque que

$$
\omega\left(x_{1}\right)=\omega(x)+\left(x_{1}-x\right) \omega^{\prime}(\xi)=\omega(x)(1+\varepsilon),
$$

$\xi$ étant compris entre $x$ et $x_{1}$, de sorte que $\varepsilon=\omega^{\prime}(\xi)$ tend vers zéro pour $x$ infini. Par suite

$$
f_{2}(x)-x=\omega(x)+\omega\left(x_{1}\right) \sim 2 \omega(x) .
$$

De même $f_{p}(x)-x$ a pour valeur principale $p \omega(x)$, ce qui conduit à penser que, pour l'itérée régulière, on aura

$$
f_{x}(x)-x \sim \alpha[f(x)-x] .
$$

(1) Il est à peine utile de rappeler que l'origine de ces formules se trouve dans un mémoire de M. Koenigs (Ann. Éc. Norm. Sup., 1884). 
Il en est nécessairement ainsi pour toute itérée assez régulière pour que sa dérivée par rapport à $x$ tende pour $x$ infini vers une limite, qui ne peut manifestement être que l'unité; car on peut dans ce cas appliquer à $f_{x}(x)$ le résultat établi pour $f(x)$; si alors $\alpha=\frac{p}{q}$, la valeur principale de $f_{p}(x)-x$ doit être égale, d'une part à $p[f(x)-x]$, d'autre part à $q\left[f_{x}(x)-x\right]$.

En comparant les formules (13) et (15), il vient

$$
\alpha=\lambda(y)-\lambda(x)=\lim _{n \rightarrow \infty} \frac{y_{n}-x_{n}}{x_{n+1}-x_{n}},
$$

formule qui définit parfaitement $\alpha$ en fonction de $x$ et $y$, et par suite $y=f_{\alpha}(x$ en fonction de $a$ et $x$.

Or il est facile de montrer que cette formule converge effectivement toutes les fois que $\omega^{\prime}(x)$ tend vers zéro d'une nanière monotone, ou même est à variation bornée de $x_{0}$ à l'infini. Désignant en effet par $\alpha_{n}$ le rapport dont on cherche la limite, on obtient par un calcul facile

$$
\alpha_{n+1}-\alpha_{n}=\alpha_{n} \frac{\omega\left(x_{n}\right)}{\omega\left(x_{n+1}\right)}\left[\omega^{\prime}\left(\xi_{n}\right)-\omega^{\prime}\left(\xi_{n}^{\prime}\right)\right]
$$

où $\xi_{n}$ et $\xi_{n}{ }^{\prime}$ sont compris entre $x_{n}$ et $x_{n+1}$. Au second membre, le premier facteur est compris entre 0 et 1 si (ce qu' on peut supposer) $y$ est compris entre $x$ et $x_{1}$; le second tend vers l'unité, et le troisième est le terme d'une série absolument convergente. La convergence de la série $\Sigma\left(\alpha_{n+1} \quad \alpha_{n}\right)$, c'está-dire l'existence de la limite de $\alpha_{m}$, en résulte. Nous avons donc établi d'une manière rigoureuse, sous la seule condition que $\omega^{\prime}(x)$ tende vers zéro et soit à variation bornée, l'existence d'une fonction itérée verifiant la relation asymptotique (15); s' il s'agit de fonctions parfaitement régulières, il est clair que c'est l'itérée ainsi définie qui est régulière. Pour toute antre détermination de $f_{x}(x)$, à cause de ce qui a été dit sur le caractère périodique de la variation relative de deux déterminations différentes, il faudrait au second membre de la relation (15) remplacer $\alpha$ par $\alpha+P[\lambda(x)], P$ désignant une fonction périodique, et l'on obtiendrait une fonction manifestement irrégulière.

Considérons maintenant le cas de fonctions $f(x)$, toujours supérieures à $x$, et de la forme $x \mathbf{Q}(x)$, la fonction $\mathbf{Q}(x)$ ne tendant pas nécessuirement vers l'unité, pouvant même devenir infinie, mais moins rapidement que n'importe quelle puissance de $x$. On est alors ramené au cas précédent en considérant $\log f(x)$ comme fonction de $\log x$ (III même changement de variable effectué sur la variable et la fonction ne changeant pas l'équation fonctionnelle de 
l'itération). Donc, sous la condition que la fonction $\boldsymbol{\Omega ( \infty )}$ soit assez régulière pour que $\frac{x \mathbf{\Omega}^{\prime}(x)}{\mathbf{\Omega}(x)}$ tende vers zéro et soit à variation bornée, on obtient une itérée, bien déterminée par la condition que $f_{\alpha}(x)$ soit de la forme $x$ Q $x+\varepsilon(x)$, $\varepsilon$ tendant vers zéro pour $x$ infini; c'est celle-là qui est régulière si la fonction $f(x)$ est régulière, et pour toute autre itérée il faudrait remplacer $\alpha-1-\varepsilon$ par $\alpha+P[\lambda(x)]+\varepsilon$, la fonction $P$ étant périodique.

Le cas de fonctions á croissance un peu plus rapide, croissant par exemple comme une puissance de $x$, se traite de même par une deuxième application du même changement de variable. Mais la portée de cette méthode est limitée; pour traiter par un changement simultané de variable et de fonction le cas ou $f(x)$ croft comme $e^{x}$, par exemple, il faudrait commatre une fonction régalière croissant plus lentement que toutes les itérées de $\log x$, et l'on ne peut précisément y urriver que par l'itération régulière de $e^{x}$ (ou de la fonction inverse $\log x$ ).

11. Il faut donc introduire des considérations d' un autre ordre. Nous allons d'abord montrer que, quelque rapide que soit la croissance des fonctions considérées (que nous supposerons toujours continues, croissantes, et supérieures à $x$, pour $x>a$ ), on peut les réunir en groupes tels qu'à chaque itérée $f_{x}(x)$ d' une fonction $f(x)$ d' un groupe, on peut, par une formule asymptotique analogue à la formule (16), faire correspondre une itérée $g_{a}(x)$ de n'importe quelle fonction du même groupe: si d'ailleurs les fonctions $f(x)$ et $g(x)$ sont régulières, à l' itérée régulière $f_{\alpha}(x)$ correspondra ainsi l'itérée régulière $g_{x}(x)$.

Nous dirons quẹ les fonctions d'un même groupe sont équivalentes au point de vue de l'ilèration.

Nous désignerons par $\lambda(x)$ et $\mu(x)$ les logarithmes d'itération correspondant respectivement aux fonctions $f(x)$ et $g(x)$, c'est-à-dire que les formules

$$
y=f_{\alpha}(x)=g_{\beta}(x)
$$

peuvent s'écrire

$$
\alpha=\lambda(y)-\lambda(x), \quad \beta=\mu(y)-\mu(x) .
$$

Nous poserons en outre

$$
\omega(x)=\lambda[g(x)]-\lambda[f(x)]=\lambda[g(x)]-\lambda(x)-1,
$$

de sorte que $g(x)=f_{1, \omega(x)}(x)$. 
Ceci posé, la condition essentielle pour que les fonctions $f(x)$ et $g(x)$ soient équivalentes au point de vue de l'itération est que $\omega(x)$ tende vers zéro pour $x$ infini, e'est-à-dire que $g(x)$ soit de la forme $f_{1+s}(x)$, $\varepsilon$ étant infiniment petit. Supposant de plus que $\omega(x)$ soit à variation bornée dans un intervalle $(\xi, \infty)$, nous allons montrer que, $f_{x}(\infty)$ étant connu, on peut déterminer la fonction 'terée $g_{\beta}(x)$ par la condition qu' elle soit de la forme $f_{\beta+\beta}(x)$, $\varepsilon$ étant infiniment petit; en d'autres termes la différence $\beta-\alpha$ des deux nombres $\alpha$ et $\beta$ definis par les formules (17) et (18) doit tendre vers zéro pour $x$ infini et $\beta$ constant; cette convergence sera même uniforme par rapport à $\beta$, pourvu que $0<\beta \leq 1$, c'est-à-dire que $x<y \leq g(x)$; si donc $x$ et $y$ augmentent indéfiniment en satisfaisant à cette double inégalité, $\beta-\alpha$ tendra vers zéro. Inversement, cette condition déterminera $f_{\alpha}(x)$ si $g_{\beta}(x)$ est connu.

Observons d'abord que cette condition conduit aisément à des formules permettant de déduire l'une de l'autre les fonctions $\lambda(x)$ et $\mu(x)$. Il suffit de remplacer $x$ et $y$ dans les formules (18), d' une part par $x_{n}=f_{n}(x)$ et $y_{n}=f_{n}(y)$, d'autre part par $X_{n}=g_{n}(x)$ et $Y_{n}=g_{n}(y)$. Dans le premier cas; $\alpha$ est indépendant de $n$, et $\beta$ prend une valeur $\beta_{n}$ qui tend vers $\alpha$ pour $n$ infini; dans le second, l'inverse a lieu, $\beta$ étant indépendant de $n$ et $\alpha$ prenant une valeur $\alpha_{n}$ qui tend vers $\beta$. On a ainsi les formules cherchées

$$
\left\{\begin{array}{l}
\alpha=\lambda(y)-\lambda(x)=\lim _{n \rightarrow \infty}\left[\mu\left(y_{n}\right)-\mu\left(x_{n}\right)\right], \\
\beta=\mu(y)-\mu(x)=\lim _{n \rightarrow \infty}\left[\lambda\left(Y_{n}\right)-\lambda\left(X_{n}\right)\right] .
\end{array}\right.
$$

Montrons maintenant, en supposant pour fixer les idées que l'on comnaisse $\lambda(x)$ et que $x<y \leq g(x)$, que la seconde de ces formules converge bien, et définit une fonction $\beta=\mu(y)-\mu(x)$ ayant bien les propriétés indiquées. On a en effet

$$
\begin{gathered}
\alpha_{n+1}-\alpha_{n}=\lambda\left[g\left(Y_{n}\right)\right]-\lambda\left(Y_{n}\right)-\lambda\left[g\left(X_{n}\right)\right]+\lambda\left(X_{n}\right) \\
=\omega\left(Y_{n}\right)-\omega\left(X_{n}\right)
\end{gathered}
$$

et

$$
x<y \leq X_{1}<Y_{1}<\ldots<X_{n}<Y_{n} \leq X_{n+1}<\ldots
$$

Il en résulte que la série de terme général $\alpha_{n+1} \cdots \alpha_{12}$ est absolument convergente, et que sa somme $\beta-\alpha$ (puisque $\alpha=\alpha_{0}, \beta=\lim \alpha_{n}$ ) est au plus égale en valeur absolue a la variation totale de $\omega(x)$ entre $x$ et l'infini. Comme de plus $\beta$ croit avec $y$ depuis 0 pour $y=x$ jusqu' a 1 pour $y=g(x)$, on a bien une fonction susceptible d'être prise pour logarithme d'itération et de définir une fonction itérée $g_{\beta}(x)$, et telle que $\beta-\alpha$ tende uniformément vers zéro quand $x$ augmente indéfiniment. 
12. Nous avons supposé que $\omega(x)$ tende vers zéro et soit à variation bornée; cette hypothèse fuit, du moins à première vue, intervenir le choix d'une itérée particuliére $f_{x}(x)$. Nous allons montrer que le résultat obtenu subsiste pour les autres fonctions itérée $F_{\alpha}(x)$ de $f(x)$, c'est-à-dire que par les formules (20) à toute itérée $F_{\alpha}(x)$ de $f(x)$ correspond une itérée $G_{\beta}(x)$ de $g(x)$, qui est de la forme $F_{x+\varepsilon}(x)$. Nous verrons ensuite que les hypothèses mêmes faites sur $\omega(x)$ sont en partie indépendantes du choix de l'itérée particulière $f_{x}(x)$.

Nous savons que, si l'on pose

$$
y=f_{x}(x)=F_{x^{\prime}}(x)
$$

la relation ainsi établie entre $\alpha$ et $\alpha^{\prime}$ (pour $x$ fixe et $y$ variable) est telle que ce deux variables croissent d'une manière continue avec $y$, la différence $\alpha-\alpha=P(\alpha)$ ne changeant pas quand $\alpha$ augmente d'une unité. Posons de même $\beta^{\prime}=\beta+P^{\prime}(\beta)$, et définissons une fonction itérée $G_{\beta^{\prime}}(x)$ de $g(x)$ par la relation

$$
g_{\beta}(x)=G_{\beta}(x)
$$

En égalant ces expressions aux précédentes, c'est-à-dire en posant

$$
y=f_{x}(x)=g_{\beta}(x)=F_{\alpha}(x)=G_{\beta}(x),
$$

nous savons que la relation ainsi établie entre $\alpha$ et $\beta$, pour $x<y<g(x)$, est telle que $\beta-\alpha$ tend vers zéro; alors $\beta^{\prime}-\alpha^{\prime}$ tend aussi vers zéro, c'est-àdire que les fonctions $F_{\alpha^{\prime}}(x)$ et $G_{\beta}(x)$ entre elles la même relation asymptotique que $f_{\alpha}(x)$ et $g_{\beta}(x)$, c. q. f. d.

Si d'ailleurs $r(x)$ et $g(x)$ sont des fonctions régulières, et si $f_{\alpha}(x)$ est l' itérée régulière de $f(x)$, la relation $\beta^{\prime}=\alpha^{\prime}-1 \varepsilon^{\prime}=\alpha+P(\alpha)+\varepsilon^{\prime}$, [ $\varepsilon^{\prime}$ tendant vers zéro et $P(\alpha)$ ètant périodique] nous montre que $\alpha$ et $\beta^{\prime}$ ne peuvent pas être des fonctions régulières d'une même variable $y$; la fonction $y=f_{\alpha}(x)$ étant régulière, la fonction $y=G_{\beta}(x)$ ne saurait être une fonction régulière de $\beta^{\prime}$. L'itérée régulière de $g(x)$ ne peut donc être que la fonction $g_{\beta}(x)$ déduite de $f_{\alpha}(x)$ par la seconde formule (20).

Revenant maintenant aux hypothèses faites sur $\omega(x)$, il est facile de voir que le fait que cette fonction tende vers zéro est indépendant du choix de la fonction itérée $f_{x}(x)$; en effet $\omega(x)$ est de la forme $\lambda(z)-\lambda(y)$, et nous avons vu au $n .^{\circ} 9$ que quand on passait d'une détermination de la fonction itérée à une autre, une expression de cette nature se trouve multipliée par un facteur limité inférieurement et supérieurement. 
On peut d'ailleurs domner a la condition que $\varepsilon=\omega(x)$ tende vers zéro une forme ne faisant pas intervenir $f_{x}(x)$. Il est commode à cet effet d'introduire les fonctions $\varphi(x)$ et $\psi(x)$ définies par

$$
g(x)=\varphi[f(x)]=f[\psi(x)],
$$

et d'observer que

$$
\varphi\left(x_{1}\right)=f_{\mathrm{E}}\left(x_{1}\right), \quad \psi(x)=f_{\mathrm{E}}(x)
$$

en posant $x_{1}=f(x)$, et $\varepsilon=\omega(x)$. Considérons alors deux nombres $x$ et $y$ et ln suite des nombres itérés $x_{n}=f_{n}(x), y_{n}=f_{n}(y)$. On. n'a pas besoin do connaître $\alpha=\lambda(y)-\lambda(x)$ pour savoir que ce nombre est très petit si $y$ est voisin de $x$, et ne change pas quand on remplace $x$ et $y$ par $x_{n}$ et $y_{n}$; dire que $\omega(x)$ devient inférieur à n'importe quel nombre positif $\alpha$, si petit soit-il, revient donc à dire que $\psi\left(x_{n}\right)$ est inférieur, pour $n$ assez grand, à $\psi\left(y_{n}\right), x$ étant quelconque et $y-x$ étant positif et arbitrairement petit; le même résultat s' applique en remplaçant $\varphi(x)$ par $\psi(x)$.

A condition d'exclure les fonctions assez irrégulières pour que $\omega(x)$ soit tantôt très petit et tantôt fini, cette fonction, ou bien tendra vers zéro, ou bien restera supérieure à un nombre fixe, et l'on peut encore très simplement caractériser le premier cas par la condition que la fonction $\varphi(x)$ ait toutes ses itérées croissant moins rapidement que $f(x)$; [au lieu de $\varphi(x)$ on peut considérer $\psi(x)$ ]; cela résulte immédiatement des expressions (23) de ces fonctions.

Supposons en particulier que ta fonction $f(x)$ soit assez rapidement croissante pour que $\frac{f(x)}{x}$ augmente indéfiniment; elle crolt alors plus vite que toutes les itérées de $a x+b$, l'itérée d'ordre $n$ de cette fonction étant équivalente à $a^{n} x$; on peut donc, du moins si la fonction $f(x)$ n'est pas trop jrrégulière, prendre pour $\varphi(x)$ ou pour $\psi(x)$ une fonction linéaire; les fonctions obtenues $g(x)=a f(x)+b$ et $f(a x+b)$ seront équivalentes à $f(x)$ au point de vue de l'itération; on peut naturellement combiner ces deux remarques et faire à la fois un changement de variable linéaire sur $x$ et un autre sur la fonction. Si $a=1$, et que le changement de variable ne consiste que dans l'addition d'une constante, il suffit même que $f(x)-x$ augmente indéfiniment.

Nous insisterons moins sur la seconde condition imposée à $\omega(x)$ d'être à variation bornée; elle est sûrement vérifiée si les fonctions $f(x), f_{\alpha}(x)$, et $g(x)$ sont régulières, car alors $\omega(x)$ est monotone. Disons seulement que si $\omega(x)$ tend assoz rapidement vers zéro, cette condition reste vérifiée quand on remplace 
$f_{x}(x)$ par une autre fonction itérée $F_{x}(x)$; il n'en est pas de même si $\omega(x)$ tend lentement vers zéro.

13. Le fait que $x$ et $f(x)$ deviennent infinis en même temps, et que par suite $x_{n}=f_{n}(x)$ augmente indéfiniment avec $n$, a joué un rôle essentiel diuns les considérations que nous venons d'exposer. Supposons maintenant que l'on ait $f(a)=a$, la fonction $/(x)$ étant toujour's continue, croissante, et supérieure $\dot{a} x$, pour $x>a$. Alors, tandis que les $x_{n}$ d'indices positifs très grands augmentent iudéfiniment, ceux d'indices négatifs très grands tendent vers $a$, et l'on peut se proposer de déterminer une itérée par des conditions de régularité asymptotique en ce point, analogues aux conditions relatives à l'allure de la fonction a l'infini que nons avons utilisées au $11 .{ }^{\circ} 10$.

Supposons d'abord $f^{\prime \prime}(t)=1$, de sorte que $f(x)-x$ est d'un ordre infinitésimal supérieur au premier par rapport à l'infiniment petit principal $x-a$. Ce cas est analogue au premier des cas étudiés au n.. 10; il existe une itérée $f_{\alpha}(x)$, bien déterminée par la condition qu'on ait, $x$ tendant vers $a$,

$$
f_{x}(x)-x \sim \alpha[f(x)-x]
$$

et on l'obtient en calculant le logarithme d'itération par la formule

$$
\alpha=\lambda(y)-\lambda(x)=\lim _{n \rightarrow-\infty} \frac{y_{n}-x_{n}}{f\left(x_{n}\right)-x_{n}}
$$

analogue à la formule (16). La convergence de cette formule est d'ailleurs assurée quand $f^{\prime}(x)$ tend vers l'unité $d^{\prime}$ une manière monotone, ou même est à variation bornée.

Si $f^{\prime}(a)$ est bien défini à droite du point $a$, et a une valeur $k>1$, on obtient de même une itérée bien définie par la condition que $f_{a}(x)$ - a soit èquivalente à $l^{\alpha}(x-a)$; des formules élémentaires s' appliquent encore quand $f(x)-a$ se comporte au point $a$ comme une puissance de $x-a$.

14. Lal question se pose maintenant de savoir si l'itérée obtenue par les formules du $n .^{\circ}$ précédent, qui supposent la régularité au voisinage du point $x=a$, est identique à l'itérée régulière à l'infini, que nous avons définie ou cherché à définir (suivant les cas), aux $n^{0 \text { s }} 9$ à 12. Pour une fonction quelconque, on ne saurait expérer qu' il en soit ainsi. Mais les considérations intuitives déjà utilisées à plusieurs reprises conduisent à penser que pour les fonctions régulières, c'est la même itèrèe qui est régulière dans lout l'inter- 
valle $(a, \infty)$, et qui par suite est asymptotiquement régulière aussi bien au point $x=a$ qu' à l'infini.

Considérons en effet la suite des nombres itérés

$$
\ldots, \quad x_{0}=x, \quad x_{1}=f(x), \ldots, \quad x_{n}=f(x), \ldots ;
$$

nous avons vu que deux déterminations distinctes ont l'une par rapport a l'autre des variations périodiques (en appelant période l'intervalle qui sépare les nombres $x_{n}$ et $x_{n+1}$ ). Une fonction itérée qui ne serait pas régulière au point $x=a$ aurait donc des oscillations périodiques; on pourrait concevoir que ces oscillations s' atténuent progressivement pour disparâ̂tre à l'infini; mais la fonction obtenue ne serait pas parfaitement régulière. On se souvient en effet qu'au début de cette étude la notion de fonction parfaitement régulière a été déduite de cette remarque $q u^{\prime}$ une fonction comme $e^{x}+e^{-x} \sin \log x$, malgré la petitesse et la lenteur de ses oscillations, n'était pas parfaitement régulière, et.qu'il existait une courbe plus régulière recoupant une infinité de fois la courbe représentative de cette fonction. Cette notion implique donc que l'itérée régulière n'ait même pas des oscillations qui s'évanouissent à l'infini ou au point $x=a$. Il faut donc admettre que c' est la même itérée qui est régulière aux deux extrémités de l'intervalle $(a, \infty)$.

Indépendamment de ces considérations idéalistes, l'exactitude de ce principe est facile à vérifier dans des cas simples. Si $f(x)=k x,(k>0)$, $f_{a}(x)=k^{x} x$, et cette fonction est régulière aussi bien pour $x$ nul que pour $x$ infini. De même, pour $f(x)=x^{k}$, fonction pour laquelle il y a lieu de distinguer les deux intervalles $(0,1)$ et $(1, \infty)$, la même itérée est régulière aux points $0,1, \infty$. Un exemple un peu moins élémentaire est celui de la fonction $f(x)=x^{2}-2$, étudiée par M. Pincherles ( $\left.{ }^{1}\right)$. En posant

$$
x=t+\frac{1}{t}
$$

on a

$$
f(x)=t^{2}+\frac{1}{t^{2}}
$$

et la fonction itérée cherchée est

$$
f_{x}(x)=t^{\beta}+\frac{1}{t^{\beta}}, \quad\left(\beta=2^{x}\right),
$$

(1) S. Pincherle, L'iterazione completa di $x^{2}-2$. Attí della R. Accademia dei Lincei, rol. XXIX (1920), pp. 329-333. 
elle est régulière aussi bien au point double $\left(^{1}\right) x=2$, qui correspond à $t=1$, qu'à l'infini. La même remarque s'applique à la fonction $f(x)=4 x+x^{2}$, qui se déduit de la précédente en ramenant le point double à l'origine.

Admettant donc la légitimité du principe énoncé, on observe que, quoique étant la conséquence naturelle de ceux admis jusqu'ici, il introduit quelque chose d'assez nouveau dans la notion de fonction régulière. Les deux premières propriétés fondamentales (constitution d'une échelle complète et monotonie des dérivées), n'impliquaient que des propriétés vérifiées pour $x$ assez grand; deux fonctions, égales pour $x$ supérieur à une certaine valeur $X$, ne devaient pas être considérées comme distinctes, et l'on ne savait pas laquelle était le prolongement naturel de la fonction définie pour les grandes valeurs de $x$. Il est clair que cette indétermination ne provenait que de l'insuffisance des deux premières propriétés en question pour caractériser d' une manière satisfaisante les fonctions régulières; le prolongement d' une courbe régulière (et sans doute analytique) ne peut pas être indéterminé. La troisième propriété, relative aux opérations analytiques définissant des fonctions régulières, faisait bien déjà disparaître cette indétermination; mais des opérations, choisies parmi un nombre fini d'opérations, et répétées un nombre fini de fois, ne peuvent pas donner toutes les fonctions régulières. La propriété introduite maintenant a une plus grande portée. Etant donnée une fonction (succession de valeurs de $x$ dont on ne sait pas le mode de définition analytique), par une opération effectuée sur cette fonction, mettant donc en évidence ses propriétés intrinsèques et non celles de l'opération par laquelle on l'a'obtenue, nous obtenons une vérification possible de la régularité, et nous voyons que la régularité implique une solidarité entre toutes les parties de la courbe représentative, depuis le point $x=a$ jusqu'à l' infini.

15. Nous sommes maintenant en mesure d'effectuer l'itération régulière de n'importe quelle fonction régulière. Parmi les fonctions croissant plus rapidement que $x$ (et à cause de la relation entre des fonctions inverses il suffit de considérer ces fonctions), le seul cas laissé de côté jusqu'ici est celui des fonctions qui, comme $e^{x}$, croissent trop rapidement pour qu' on puisse leur appliquer les formules du $1 .^{\circ} 10$, et auxquelles on ne peut pas appliquer les méthodes du $11 .^{\circ} 13$ parce que l' équation $f(x)=x$ n' a pas de racine. Mais on pourra tolıjours, et même d'une infintié de manières, trouver une fonction

(1) Nous entendons par point double les points $x, y$ pour lesquels $y=x=f(x)$; ces points sont les points limites de $f_{n}(x)$, soit pour $n$ infini positif, s.jit pour $n$ infini négatif. 
régulière $g(x)$, équivalentè à $f(x)$ au point de vue de l'itération, à laquelle on pourra appliquer les procédés du $1 .^{\circ} 13$; l'itérée régulière $g_{x}(x)$ de $g(x)$ étant connue, on en déduira celle de $f(x)$ par la première formule (20) du n. ${ }^{\circ} 11$.

Précisons la manière dont on peut former $g(x)$, en supposant que $f(x)-x$ augmente indéfiniment, c' est-à-dire en n'excluant que des cas où la formule (16) s' npplique directement à $f(x)$. A cause de la régularité de $f(x)$, cette hypothèse implique $f^{\prime}(x)>1$ pour $x>\xi$. Choisissant alors une valeur $t>\xi$, nous prendrons pour $g(x)$ l'expression

$$
g(x)=f(x+b)-f(\ell)
$$

qui représente bien une fonction continue, croissante, et supérieure á $x$ pour $x$ positif; pour $x=0$, cette fonction est nulle et sa dérivée a la valeur $h=f^{\prime}(t)>1$. Dans ces conditions, d'après la remarque finale du $n . \circ 13$, on définit le logarithme d'itération relatif à cette fonction par la formule

$$
\mu(y)-\mu(x)=\lim _{n \rightarrow-\infty} \frac{\log g_{n}(y)-\log g_{n}(x)}{\log k}
$$

qui n'est autre que la formule (24), où l'on a remplaçé $x, \lambda, f$ par $\log x, \mu$, $\log g$. On obtient ensuite $\lambda(y)-\lambda(x)$ par la formule (20).

Cette méthode revient, en termes géométriques, $\dot{a}$ ramener le point $t, f(l)$ à l'origine par une translation; la droite ramenée sur la première bissectrice se trouve donc être la parallèle à cette bissectrice passant par le point en question. Il est peut-être plus naturel de considérer la tangente en ce point, et de la ranener sur la première bissectrice par des changements linéaires convenables effectués sur $x$ et $y$. En effectuant par exemple la même translation que précédemment, et faisant un changement d'unité soit sur $x$, soit sur $y$, cela conduit à prendre pour $g(l)$ l'une des expressions

$$
f\left[t+\frac{x}{f^{\prime}(t)}\right]-f(l), \text { ou } \frac{f(x+t)-f(l)}{f^{\prime}(t)} .
$$

$D^{\prime}$ ailleurs, d'après le $n .^{\circ} 12$, les changements linéaires effectués sur $x$ et sur $y$ sont légitimes si $\frac{f(x)}{x}$ augmente indéfiniment, c' est-à-dire en n' excluant que des cas où l'itération régulière est définie par la formule (16) appliquée, soit directement à $f(x)$, soit à $\log f(x)$ considéré comme fonction de $\log x$. Dans ces conditions la régularité implique que $f^{\prime \prime}(x)$ soit positif dès que $x$ dépasse une certaine valeur $\xi$; si alors on prend $t>\xi$, et si l'on prend pour définir $g(x)$ une des expressions (27), on a une fonction à dérivée seconde 
positive pour $x$ et tangente à l'origine à la première bissectrice. On définira alors $\mu(x)$ par la formule (24), puis $\lambda(x)$ par la formule (20).

Nous avons bien ainsi défini dans tous les cas l'itération régulière des fonctions régulières. Il est à remarqner que la régularité parfaite, qui n'est pas nécessaire pour l'application des formules asymptotiques du $1 .^{\circ} 10$, l'est pour l'application des formules du présent $n .^{\circ}$, la régularité parfaite de $f(x)$ étant équivalente à celle de la fonction $g(x)$ définie par l'une des expressions (27). Mais une fois qu'on a défini l'itérée régulière d'une fonction régulière $f(x)$, la formule (20) permet, pour toutes les fonctions équivalentes à $f(x)$ au point de vue de l'itération, même si elles ne sont pas parfaitement régulières, de définir l'itérée asymptotiquement régulière. Le champ d'application de nos formules asymptotiques, même pour les fonctions rapidement croissantes, est donc finalement bien plus étendu que celui dos fonctions régulières.

16. Les résultats qui précèdent comportent la possibilité d'un grand nombre de vérifications, à défaut d'une démonstration générale établissant la compatibilité des différents principes intuitifs utilisés, et par suite l'existence d'un ensemble de fonctions régulières ayant toutes les propriétés indiquées.

Il peut d'abord arriver que les procédés du $n 0^{\circ} 15$ s' nppliquent à une fonction $f(x)$ dont on connaît déjà l' itérée régulière par les formules élémentaires du $1^{\circ} .10$; tel est le cas pour les fonctions croissant comme une puissance de $x$. Il s'agit alors de démontrer qu' on retrouve bien la même itérée régulière par les formules du $11^{\circ} 15$; cela revient à montrer que, pour la fonction auxiliaire $g(x)$ que l'on utilise, à laquelle on peut appliquer d̀ la fois les formules asymptotiques du $n .^{\circ} 10$ relatives aux grandes valeurs de $x$, ou celles du $11 .: 13$ relative au point $x=a$ ( $a$ étant nul pour les fonctions considérées au n. 15), ces deux formules conduisent au même résultat. Nous avons déjà effectué cette vérification dans des cas particuliers.

Considérons d'autre part le cas où l'on ne dispose pas d'autres procédés que ceux du n. ${ }^{\circ} 15$, ou du moins où l'on n'utilise que ces procédés. Il s'agit alors de montrer que le résuitat est indépendant du choix de la fonction auxiliaire $g(x)$; que l'on prenne l'une on l'autre des expressions (25) et (27), que l'on donne une valeur ou une autre au paramètre auxiliaire $t$, que même l'on prenne des expressions différentes, ou doit trouver le même résultat. Ainsi, dans le cas de la fonction $f(x)=e^{x}$, on peut prendre comme fonction auxiliaire $e^{x}-1$; mais on peut aussi prendre comme fonction auxiliaire $e^{x}-e^{-x}$ ou encore $\operatorname{sh} x$. 
En principe, pour les fonctions régulières auxquelles les formules élémentaires du n. 10 ne s'appliquent pas, nous définirons l'itérée régulière eu prenant l'une des fonctions auxiliaires définies par les formules (27); les deux procédés sont sârement équivalents, car si $g(x)$ a pour fonction itérée $g_{\alpha}(x), k g\left(\frac{x}{k}\right)$ a pour fonction itérée $k g x\left(\frac{x}{k}\right)$, et, $\frac{g(x)}{x}$ augmentant indéfiniment, ces fonctions sont équivalentes au point de vue de l'itération; elles conduisent done à la même définition de $f_{\alpha}(x)$. Mais il reste à vérifier que le résultat est indépendant du choix du paramétre $t$.

Cette vérification est immédiate dans deux cas particuliers; le premier est celui où $f(x)=e^{x}$; alors la fonction auxiliaire définie par la seconde formule (27) est $e^{x}-1$; elle est indépendante de $t$. Le second cas est celui où $f(x)=a x^{2}+2 b x+c$; on peut d'abord faire disparaître le terme du premier degré en ajoutant une même constante à $x$ et $y$; en appliquant ensuite le seconde formule (27), on trouve comme fonction auxiliaire $x+\frac{x^{2}}{2 l}$, qui est de la forme $\operatorname{tg}\left(\frac{x}{t}\right)$; son itérée est donc de la forme $\operatorname{tg}\left(\frac{x}{t}\right)$, et, comme il s'agit de fonctions croissant comme des puissances de $x$ (l'exposant étant lié à $\propto$ ), un changement du paramétre $t$ est négligeable à côté $\mathrm{d}^{\prime}$ un changement de cet exposant; tontes ces fonctions sont équivalentes au point de vue de l'itération; elles conduisent nécessairement à la même itérée $f_{\alpha}(x)\left({ }^{1}\right)$.

17. L'itération régulière nous domne un nouveau procédé pour définir des fonctions régulières. Le cas où $f(x)=e^{x}$ est particulièrement important; la fonction $e_{x}^{x}$ obtenue par l'itération régulière de la fonction exponentielle comble des lacunes importantes dans l'échelle des fonctions régulières que l'on peut obtenir par les opérations régulières élémentaires. Toutes celles de ces fonctions qui augmentent indéfiniment sont en effet de la forme $e_{\omega(x)}^{x}$, la fonction $\omega(x)$ tendant vers un nombre entier. Donnant alors à $\alpha$ des valeurs comprises

(1) Il est à remarquer que, dans le cas du polynome du second degré, le point de vue elémentaire nous apprend que pour $x$ infini

$$
\log f_{x}(x) \cos 2 x \log x
$$

et les considérations du texte, montrant que l'itérée déduite de la fonetion auxiliaire $g(x)$ est indépendante de $t$, ne prouvent pas d'une manière rigoureuse que ce soit la mème que par les formules elementaires. Il faudrait montrer que pour $g(x)=x+\frac{x^{2}}{2}$, c'est la même itérée qui est régulière à l'origine et à l'infini. 
entre 0 et 1 , on obtient des fonctions $e_{\alpha}^{x}$ comblant une large lacune dans l'échelle des croissances entre les fonctions de croissance algébrique (ou telles que $\log y$ croisse comme une puissance de $\log x$, ou $\log \log y$ comme une puissance de $\log \log x$, et ainsi de suite) et les fonctions de croissance exponentielle. D' autre part $e_{x}^{c}$ et $e_{x}^{x}$ (c étant une constante) sont des fonctions régulières croissant plus rapidement que toutes les itérées d'ordre entier de $e^{x}$. On a ainsi placé de nouvelles fonctions, a la fois par extrapolation et par intrapolation, dans l'ensemble des fonctions régulières connues.

Désignons par $\Phi$ l'opération fonctionuelle

$$
\Phi[f(x) ; x]=f_{x}(x) .
$$

En la répétant, on obtient de nouvelles fonctions de plus en plus rapidement croissantes $\Phi^{p}\left[e^{x} ; x\right]$. Pour obtenir des fonctions encore plus rapidement croissantes, et qui soient régulières, il faudrait réaliser l'iuterpolation régulière de la suite des nombres obtenus en domnant a $p$ des valeurs entières, et remplacer $p$ par $x$. Au point de vue théorique, cette interpolation régulière est possible, d'aprés le $n .^{\circ} 9$. Mais pour la réaliser effectivement, il faudrait effectuer une étude asymptotique beaucoup plus difficile que dans le cas de l'itération simple, et l'on n'aurait fait que reculer la difficulté. Nous savons en effet qu'en cherchant à définir l'ensemble des fonctions régulières par la formation successive de nouvelles fonctions, il n'est pas possible d'échapper aux difficultés du transfini.

18. Une définition intrinsèque de la régularitè présenterait bien plus d'intérêt. Or les considérations du $10^{\circ} 15$ semblent bien conduire à une telle définition, et c'est sur ce résultat que je voudrais surtout attirer l'attention.

Dans les cas oủ nous n'avons pu définir l'itérée régulière que par application des procédés exposés au $n .^{\circ} 16$, nous nous trouvons. avoir te choix entre un grand nombre de définitions possibles. Pour les fonctions parfaitement régulières, il y a, comme nous l'avons exposé, bien des raisons de penser que toutes ces définitions conduisent au même résultat; on ne peut au contraire pas penser qu'il en serait de même pour les autres fonctions. On a douc bien probablement ainsi des propriétés caractéristiques des fonctions régulières.

En prenant, pour fixer les idées, le cas où l'on peut prendre les expressions (27) pour la fonction auxiliaire $g(x)$, e' est-à-dire celui où $\frac{f(x)}{x}$ augmente indéfiniment, on obtient le résultat suivant: une condition nécessaire de la 
régularité est que $f^{\prime}(x)$ soit monotone quand $x$ dépasse une certaine valeur $a$; en augmentant au besoin $a, f^{\prime}(x)$ sera positif et croissant pour $x>a$. Choisissons alors arbitrairement une valeur $t>a$, et posons

$$
\left\{\begin{aligned}
g(x) & =\frac{f(x+t)-f(t)}{f^{\prime}(t)} \\
\mu(y) \quad \mu(x) & =\lim _{n \rightarrow-\infty} \frac{g_{n}(y)-g_{n}(x)}{g_{n+1}(x)-g_{n}(x)} \\
\alpha=\lambda(y)-\lambda(x) & =\lim _{n \rightarrow \infty}\left\{\mu\left[f_{n}(y)\right]-\mu\left[f_{n}(x)\right]\right\}
\end{aligned}\right.
$$

On obtient en général pour $\alpha$ une valeur qui dépend de la valeur choisie pour $t$, et les considérations développées aux $n 0^{0 \text { s }} 14$ à 15 conduisent à penser que, pour les fonctions régulières, $\alpha$ est indépendant de $t$. Cette propriété semblant assez restrictive, on peut penser qu'il est possible de définir la régularité de $\mathrm{f}(\mathrm{x})$ par le fait que celte limite ne dépende pas de $\mathrm{t}$.

On se rappelle que nous avons été conduits à ce résultat par l'idée que la fonction $g(x)$ était régulière en même temps que $f(x)$, et qu'étant régulière de zéro à l'infini, elle avait une itérée régulière aussi bien pour $x$ nul que pour $x$ infini. Une remarque tout à fait différente apparait comme une confirmation de cette idée: c'est que, dans toute autre hypothèse que celle où $\alpha$ ne dépend pas de $t$, cette quantité est de la forme $\lambda(y, l)-\lambda(x, l)$, cette fonction $\lambda$ étant manifestement irrégulière. En effet, sa partie entière ne pouvant donner lieu à aucune ambiguité, ou bien $\alpha$ tend vers une limite $\lambda(y)-\lambda(x)$ quand $t$ augmente indéfniment, ou bien elle oscille indéfiniment entre deux valeurs limites différentes. Dans ce dernier cas l'irrégularité est évidente; dans le premier, à cause de la périodicité des variations relatives de deux déterminations différentes du logarithme d'itération, $\alpha(l)=\lambda(y, t)-(x, l)$ tend vers $\alpha=\lambda(y)-\lambda(x)$ par valeurs tantôt plus petites, tantôt plus grandes; pour fixer les idées, si $\alpha$ étant constant, on fait augmenter $x$ indéfiniment, $y$ devient une fonction $\mathrm{f}_{x}(x)$ de $x$, et $\alpha(t)$ devient une fonction de $x$ et $t$ qui, pour $t$ intini, tend vers $\alpha$ par valeurs plus petites on plus grandes suivant la valeur de $x$; il y a ainsi une infinité d'oscillations quand $x$ augmente indéfiniment. De toute façon, à moins que $\alpha$ soit indépendant de $t$, les formules (28) conduisent à une fonction irrégulière, et il est naturel de penser que, les opérations effectuées apparaissanl comme régulières, ces irrégularités prouvent l'irrégularité de $f(x)$. Sans doute ce raisonnement est-il discutable, mais il est intéressant de noter que des considérations intuitives assez différentes les unes des autres conduisent au même résultat. 
La définition de la régularité étant ainsi précisée quand $\frac{f(x)}{x}$ augmente indéfiniment, on peut aisement définir la régularité dans les autres cas: si $f(x)$ augmente indéfiniment mais non $\frac{f(x)}{x}$, la fonction $f(x)$ sera régulière si le produit $x f(x)$ est régulier ( $\left.{ }^{1}\right)$; si $f(x)$ tend vers $-\infty$, la fonction $f(x)$ sera régulière si $-f(x)$ l'est; si enfin elle n'augmente pas indéfiniment, elle sera régulière si elle tend vers une limite $c$ et si $\frac{1}{f(x)-c}$ est régulier. La régularité est ainsi définie dans tous les cas.

Telle est la définition que nous proposons; nous y avons été conduit par des considérations intuitives. Mais on peut se proposer de prendre cette définition comme point de départ, et de montrer que l'ensemble des fonctions régulières ainsi définies a bien les propriétés considérées au début de cette étude, et notamment qu'il constitue une échelle complète, que ces fonctions sont continues et monotones pour $x$ assez grand, et que la régularité se conserve par les opérations régulières élémentaires. Il y a là un champ de recherches qui paraît fort difficile; mais même un résultat partiel peut presénter de l'intérêt.

Il nous reste, pour montrer l'importance pratique de cette théorie, à indiquer deux applications.

\section{CHAPITRE III.}

\section{Applications.}

19. La première est relative à la sommation des séries divergentes, ou, ce qui revient au même, à la définition de la limite généralisée d'une fonction $u(x)$ d' une variable $x$ indéfiniment croissante; nous supposerons cette fonction bornée. Si elle n'a pas de limite, il peut exister une limite généralisée définie par l'une des expressions

$$
\begin{gathered}
\lim _{X \rightarrow \infty} \frac{1}{X} \int_{x_{0}}^{X} u(x) d x \\
\lim _{X \rightarrow \infty} \frac{1}{f(X)} \int_{x_{0}}^{X} f^{\prime}(x) u(x) d x,
\end{gathered}
$$

(1) Peut être serait.il plus naturel de n'adopter cette définition que si $f(x)>x$; si $f(x)<x$, la fonction $f(x)$ serait régulière en mème temps que la fonction irverse. 
la fonction $f(x)$ augmentant indéfiniment. D'ailleurs, si la première de ces formules est applicable, la deuxième s'applique a fortiori et dome la même limite toutes les fois que $f(x)$ est une fonction inférienre $\dot{a} x$, et monotone ainsi que sa dérivée $f^{\prime}(x)$. Mais si la formule (29) ne donne pas une limite déterminée, on peut en obtenir une par la formule (30), et l'on a d'autant plus de chances d'en obtenir une qu'on a pris pour $f(x)$ une fonction plus lentement croissante.

La question se pose alors de savoir si l'on ne risque pas d'obtenir deux limites différentes en prenant deux fonctions différentes

$$
y=f(x) \quad \text { et } \quad z=g(x)=\varphi(y)=\varphi[f(x)] .
$$

Or le remplacement de $y$ par $z$ par la formule $z=\varphi(y)$ est analogue à celui de $x$ par $y=f(x)$ qui fait passer de la formule (29) à la formule (30). Il en résulte que l'on ne risquera pas d'avoir des résultats contradictoires si $\varphi(y)$ est une fonction inférieure à $y$, augmentant indéfiniment, et monotone airsi que sa dérivée. Ce résultat est bien commu. Au contraire, si $y$ et $z$ ont des irrégularités relatives sensibles, si par exemple

$$
z=g(x)=\int_{0}^{X} f^{\prime}(\xi)\left[1-\frac{1}{2} \sin ^{2} f(\xi)\right] d \xi,
$$

on voit aisément que les fonctions $f(x)$ et $g(x)$, mises dans la formule (30), peuvent donner des résultats différents; tel est le cas si $u(x)=\sin ^{2} f(x)$.

On expliquera sans doute cette circonstance en disant qu' une des fonctions $f(x)$ et $g(x)$ est irrégulière et ne convient pas comme fonction sommatrice. Mais la difficulté est de savoir laquelle. Dans un Mémoire publié en 1926 dans le Bulletin de la Société Mathématique, j'ai montré que la difficulté existe surtout pour les fonctions à croissance lente comme $\log \log x$, ou a fortiori pour celles qui croissent plus lentement encore. Ainsi en prenant

$$
f^{\prime}(x)=\frac{1}{x \log x}, \quad g^{\prime}(x)=\frac{1}{x \log x}\left(1-\frac{1}{2} \sin ^{2} \log \log x\right),
$$

on obtient des fonctions $f(x)$ et $g(x)$ monotones, pour $x$ assez grand ainsi que toutes leurs dérivées. Sans doute, dans ce cas particulier, nous savons que nous devons considérer que $f(x)$ est régulier et non $g(x)$. Mais cet exemple nous montre que même des fonctions dont toutes les dérivées sont monotores pour $x$ assez grand ne sont pas toujours assez régulières pour être utilisées comme fonctions sommatrices. Le problème de trouver une condition suffisante pour qu'il en soit ainsi, cette condition ne fuisant intervenir que la régularité 
des fonctions considérées et s'appliquant même aux fonctions à croissance, très lente, est un probléme difficile, non résoluble par des procédés élémentaires.

Je n'avais pas sans doute été assez clair dans la rédaction de mon Mémoire de 1926, car, peu après sa publication, M. le Professeur E. BorTolotTi, rappelant un résultat publié par lui en 1921, disait avoir résolu d' une maniére élémentaire le problème posé. Mais les conditions imposées par lui à $f(x)$ excluent le cas des fonctions lentement croissantes; c'est précisément dans ce cas que j'avais voulu poser le problème, sachant qu'il n'est difficile que dans ce cas, et qu'il ne faut pas écarter ce cas si l'on veut une formule de sommation applicable dans des conditions aussi larges que possible.

Or ce problème, non résolu jusqu'ici, l'est par la théorie des fonctions parfaitement régulières. $\mathrm{Si}$ ell effet $y$ et $z$ sont deux fonctions régulières de $x$, indéfiniment croissantes, et si pour fixfr les idées $y>z$, on a $z=\varphi(y)$, la fonction $\varphi$ étant régulière, et par suite monotone ainsi que sa dérivée; si alors on obtient une limite en prenant $y=f(x)$ comme fonction sommatrice, le résultat rappelé ci dessus s'applique et l'on obtient la même limite en.prenant $z=\varphi(y)=g(x)$.

On ne risque donc pas d'obtenir de contradiction en appliquant la formule (30) avec différentes fonctions régulières $f(x)$.

20. L'autre application est liée à ce que j'ai appelé une correspondance homéomorphe dans mon Mémoire déjà cité sur les échelles complètes de croissance. C' est une correspondance biunivoque entre les fonctions $f(x)$ de la première échelle, de croissances intermédiaires entre celles de deux fonctions domnées $f_{t}(x)$ et $f_{2}(x)$, et les fonctions $g(x)$ de la seconde échelle, intermédiaires entre deux fonctions $g_{4}(x)$ et $g_{2}(x)$; de plus a des fonctions $f(x)$ de plus en plus rapidement croissantes correspondent des fonctions $g(x)$ de plus en plus rapidement croissantes. Cette correspondance biunivoque est analogue à celle qui existe entre deux variables $x$ et $y$ quand l'une est une fonction continue et croissante de l'autre; la possibilité de l'inversion résulte du principe de continuité.

Le cas de la correspondance homéomorphe entre deux fonctions régulières est particulièrement important.

Considérons, pour fixer les idées, la relation

$$
f(x)=\int_{0}^{\infty} \varphi(x, y) g(y) d y .
$$

Si la fonction $\varphi(x, y)$ est régulière, la régularité de $g(y)$ entraîne celle de $f(x)$, 
comme nons l'avons déjà observé au $11 .^{\circ} 5$. Si de plus $\varphi(x, y)$ est positif, et si, pour $Y>y$, le rapport $\frac{\varphi(x, Y)}{\varphi(x, y)}$ augmente indéfiniment avec $x$, à des fonctions $g(x)$ de plus en plus rapidement croissantes correspondent des fonctions $f(x)$ de plus en plus rapidement croissantes, le signe de $f_{1}(x)-f_{2}(x)$ pour $x$ très grand étant celui de $g_{1}(x)-g_{2}(x)$. La question se pose alors de savoir si à toute fonction régulière $f(x)$ correspond une fonction $g(x)$.

S'il n' en est pas ainsi, les tonctions régulières $g(x)$ se divisent en denx catégories, suivant que l'intégrale

$$
\int_{0}^{\infty} \varphi(x, y) g(y) d y
$$

crô̂t plus vite ou moins vite que $f(x)$, et à la fonction $f(x)$ correspondrait ainsi, non une fonction $g(x)$ déterminée, mais une coupure dans l'ensemble des fonctions régulières; on peut dire que cette coupure définit une fonction idéale $\mathcal{G}(x)$.

In existe effectivement des fonctions idéales de cette nature; ainsi on définit une fonction idéale séparant les fonctions $g(x)$ qui restent fiuies de celles qui augmentent indéfiniment. Mais il semble bien que ces fonctions idéales ne risquent pas d'être confondues avec les fonctions véritables; que notamment une coupure correspondant à une fonction véritable soit caractérisée par cette propriété que l'on peut trouver deux fonctions sèparées par cette coupure dont la différence tende vers zéro plus rapidement que n'importe quelle fonction donnée. On peut alors dire en termes peu précis que toute coupure qui semble ne pas présenter les caractères d'une fonction idénle définit une fonction véritable.

Je ne peux que renvoyer a mon mémoire cité le lecteur désireux d'avoir une idée plus précise des difficultés que soulève cette question. A défaut de solution générale pour les échelles de croissance que j'ai appelées normales, on peut chercher à les résoudre pour les fonctions régulières. Il en résulterait alors que l'équation (31) définit une correspondance homéomorphe et peut être résolue par rapport à $g(x)$, la solution étant unique dans le champ des fonctions régulières.

Le même principe de continuité, s'il était établi d'une manière satisfaisante, montrerait l'existence et l'unicité, daus le champ des fonctions régulières, des solutions. d'équations fonctionnelles trés générales, comprenant en particulier celle qui défnit l'itérée d'ordre rationnel domé d'une fonction domée. 